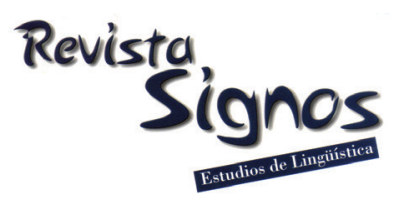

\title{
Reconocimiento visual y categorización semántica de palabras nuevas en inglés como lengua extranjera (L2): El rol de la lectura y las actividades explícitas de vocabulario*
}

\author{
Visual recognition and semantic categorization of novel words in \\ English as a foreign language (L2): The role of incidental reading \\ and vocabulary exercises
}

Andrea Rodríguez Morán

Universidad Católica de la Santísima Concepción Chile

andrea.rodriguez37@gmail.com
Roberto A. Ferreira

Universidad Católica de la Santísima Concepción Chile rferreira@ucsc.cl

Recibido: 22-X-2014 / Aceptado: 24-IV-2015

\section{Resumen}

En el presente estudio experimental se investigó el efecto de la lectura incidental (LI) y la lectura acompañada de ejercicios de vocabulario (LEV) en el aprendizaje léxico en inglés como lengua extranjera (L2). La muestra estuvo constituida de 20 alumnos de sexto básico cuyo nivel de inglés correspondía a pre-intermedio. El experimento adoptó un diseño de medidas repetidas y se llevó a cabo durante tres días consecutivos, en los que se realizaron dos sesiones de aprendizaje y una sesión de evaluación. Los resultados demostraron que las palabras aprendidas a través de la condición LEV tuvieron mayor precisión y tiempos de reacción más rápidos, tanto en la tarea de reconocimiento visual como en la de categorización semántica. Lo anterior sugiere que la lectura en combinación con actividades explícitas de vocabulario benefician tanto la adquisición de la forma léxica como del significado, por sobre la lectura puramente incidental.

Palabras Clave: Reconocimiento visual, categorización semántica, aprendizaje léxico, lectura incidental, ejercicios de vocabulario. 


\begin{abstract}
This experimental study investigated the effects of incidental reading and reading plus vocabulary exercises on the learning of novel words in English as a foreign language (EFL). The participants were a group of 20 pre-intermediate EFL learners in 6th grade. The experiment took place over three consecutive days and used a repeated-measures design that included two learning sessions and one testing session. The results showed that novel words learned in the reading plus vocabulary exercises condition had higher accuracy and faster reaction times than those learned in reading only, both in visual word recognition and semantic categorization. This suggests that the combination of reading and explicit vocabulary activities benefits both the acquisition of word form and meaning, beyond incidental reading alone.
\end{abstract}

Key Words: Visual word recognition, semantic categorization, word learning, incidental reading, vocabulary exercises.

\title{
INTRODUCCIÓN
}

La adquisición del lenguaje es el proceso mediante el cual un individuo desarrolla su lengua materna o cualquier otra lengua a la cual esté expuesto durante la vida. Este proceso parece tener lugar de manera relativamente rápida debido a que alrededor de los tres años un niño ya es capaz de formar y comunicar ideas básicas (Lew-Williams \& Fernald, 2007; Bannard \& Matthews, 2008; Rakoczy, Warneken \& Tomasello, 2008). Sin embargo, la adquisición léxica no termina allí, sino que se prolonga a lo largo de toda la vida tanto para un hablante de lengua materna como para un hablante de lengua extranjera.

La educación formal contribuye a este proceso exponiendo a los hablantes a miles de palabras nuevas a través de la lectura de textos escolares. De hecho, durante los años de escolaridad una persona está expuesta a más de 88 mil familias de palabras y aproximadamente aprende la mitad de estas al término de la vida escolar, es decir, cerca de 3 mil palabras por año (Nagy \& Anderson, 1984; Nagy \& Townsend, 2012). De estos datos se desprende que la mayoría de las palabras aprendidas no proviene de un programa de lectura para el aprendizaje léxico, ya que sería imposible enseñar tal cantidad de palabras de manera directa en un año (Grabe, 2008). En realidad, Stahl (1991) estima que es posible presentar solo alrededor de 300 a 400 palabras durante el curso de un año escolar, lo que discrepa ampliamente con el número propuesto por Nagy y colegas. Si la inmensa cantidad de palabras que los niños aprenden durante su desarrollo no se enseña de forma sistemática en la escuela, una de las posibilidades para alcanzar tan elevadas cifras es adquirir nuevas palabras en contexto de manera incidental a través de la lectura. Se sabe que este proceso ocurre de manera espontánea, incluso en una lengua extranjera (Rashidi \& GanbariAdivi, 2010; Vidal, 2011; Ahmad, 2012), por lo que se puede afirmar que la lectura incidental, en conjunto con otras fuentes de estímulos lingüísticos, sin duda facilitan 
la tarea del aprendizaje de palabras nuevas tanto en la lengua materna como en la lengua extranjera (Kuppens, 2010). Cabe mencionar sí que el aprendizaje léxico en L2 supone una desventaja comparativa de estímulos externos a la lectura, ya que en la práctica este proceso se limita principalmente a la enseñanza dentro de la sala de clases. Por esta razón, el contexto escrito entregado por la lectura juega un rol aún mayor en el proceso de aprendizaje léxico en L2. En esta línea, se ha sugerido que el impacto que la lectura incidental tiene en el aprendizaje léxico podría incrementarse de manera significativa si esta se acompaña de definiciones o ejercicios de vocabulario que permitan trabajar de manera explícitas las palabras desconocidas (Zimmerman, 1997; Paribakht \& Wesche, 1999; Sonbul \& Schmitt, 2010).

Los estudios citados anteriores han permitido reunir suficiente evidencia para avalar que existe un efecto estable a favor de la lectura acompañada de actividades explícitas. No obstante, es importante notar que estas investigaciones no han ofrecido explicaciones rigurosas sobre los procesos cognitivos inherentes al aprendizaje y al acceso léxicos. Por ejemplo, no se ha propuesto un modelo que explique la forma en que el tipo de aprendizaje (incidental o explícito) influencia el proceso de acceso al léxico nuevo en L2. Otro aspecto menos relevante pero que igualmente es importante señalar es el poco control que ha habido en la selección de las muestras, ya que en la mayoría de los casos, los participantes eran hablantes de inglés L2 pero de culturas y lenguas maternas distintas. Estos antecedentes dan pie para proponer un trabajo que controle con mayor eficacia las variables lingüísticas y psicolingüísticas.

En el presente estudio se busca investigar de manera experimental el efecto de la lectura, con y sin ejercicios de vocabulario, en el aprendizaje léxico en inglés L2. En particular, se intenta explicar de qué forma la lectura incidental y la lectura con ejercicios de vocabulario afectan el acceso a la forma léxica y al significado de las palabras adquiridas recientemente. Se propone que ambas condiciones son motores del aprendizaje léxico; sin embargo, la lectura acompañada de ejercicios de vocabulario produce mayor retención, permitiendo así un mejor acceso al léxico. Por otra parte, con el fin de tener un mejor control de las variables extrañas y mayor precisión en las medidas, en la presente investigación los participantes provienen de un mismo país y tienen como lengua materna el español. A diferencia de los estudios previos sobre aprendizaje léxico en L2, los cuales revisaremos en detalle en el marco teórico, en este trabajo no solo se mide el porcentaje de respuestas correctas, sino también los tiempos de reacción (TRs) durante el procesamiento léxico.

\section{Marco teórico}

Es importante destacar que, en términos educacionales, el aprendizaje léxico a través de la lectura puede variar dependiendo de la metodología utilizada, ya que en algunos casos el objetivo es lograr que los aprendices utilicen el contexto escrito como fuente para derivar el significado, y como resultado, aprender palabras nuevas. 
En otros casos, no existe ningún objetivo explícito de aprendizaje léxico, lo que corresponde a un aprendizaje incidental de vocabulario. A pesar del rol protagónico de la lectura como fuente de aprendizaje léxico, esta tiene algunas desventajas tales como la lentitud del proceso y la dificultad de predecir qué palabras exactamente serán aprendidas con posterioridad a la exposición de un texto (Paribakht \& Wesche, 1997; Webb, 2008).

Algunos de los factores más importantes que intervienen en la adquisición del vocabulario, ya sea a través de la lectura incidental o con la utilización de otros métodos, son la frecuencia de aparición de las palabras, la calidad del contexto y la riqueza semántica. Numerosos estudios han demostrado que, a mayor número de exposiciones, mayor es la retención de vocabulario (Rott, 1999; Webb, 2007; Petchko, 2011). Es preciso notar que en otros estudios se ha demostrado que el número de apariciones de una palabra favorece principalmente el aprendizaje de su ortografía, mientras que el significado depende principalmente de la calidad del contexto donde la palabra se encuentra inserta (Webb, 2008). Por otra parte, en estudios de inglés como lengua materna, se ha descubierto que la variedad de contextos o diversidad contextual, una vez controlada la frecuencia de aparición, también tiene un efecto en el aprendizaje léxico en diversas tareas lingüísticas (Bolger, Balass, Landen \& Perfetti, 2008; Ferreira, 2011). En otras investigaciones se ha descubierto que la diversidad contextual de una palabra puede predecir el tiempo de reacción en tareas de reconocimiento visual de mejor forma que la frecuencia (Adelman, Brown \& Quesada, 2006; Perea, Soares \& Comesaña, 2013). Otra variable que afecta el aprendizaje y el procesamiento léxicos es la riqueza semántica. Las palabras que poseen un gran número de atributos semánticos se procesan de manera más rápida en tareas de decisión léxica y categorización semántica (Pexman, Siakaluk \& Yap, 2013). Este efecto también se ha observado en estudios de aprendizaje léxico donde las palabras nuevas asociadas a un mayor número de atributos semánticos durante las etapas de aprendizaje muestran mejor precisión y tiempos de reacción más bajos en la tarea de categorización semántica (Ferreira, Göbel, Hymers \& Ellis, en prensa).

Si bien se acepta que la lectura incidental por sí sola es suficiente para incorporar los diferentes factores descritos anteriormente y, de esta forma, hacer posible el aprendizaje léxico, también es necesario señalar que el efecto de la lectura en el aprendizaje puede incrementarse de manera significativa si se acompaña de elementos adicionales, tales como, definiciones o ejercicios de vocabulario tendientes a clarificar el significado de las palabras desconocidas (Paribakht \& Wesche, 1999; Sonbul \& Schmitt, 2010). Esta propuesta contrasta con la idea de una lectura puramente incidental y abre una nueva perspectiva de aproximación al aprendizaje del léxico. El hecho de incorporar definiciones o ejercicios durante la lectura o después de esta necesariamente involucra estar consciente del aprendizaje de palabras específicas, lo cual no ocurre en la lectura incidental. 
Jenkins, Matlock y Slocum (1989) realizaron una de las investigaciones pioneras en L1 que permitieron observar el efecto de las estrategias de derivación de significado a través del contexto y la enseñanza directa de significados. Los resultados de este estudio demostraron que los individuos (alumnos de quinto año de primaria) que recibieron una instrucción directa del significado aprendieron en todas las condiciones de práctica (baja, media y alta), mientras que los individuos que accedieron a las palabras a través del contexto lograron un avance solo en condiciones de práctica media y alta. Esto da a entender que, si bien los individuos pueden aprender palabras desconocidas en ambas condiciones, la instrucción directa y explícita del significado parece ser más efectiva que la presentación de este a través del contexto.

Los resultados de estudios en L1 en gran medida han sido replicados en investigaciones posteriores en L2. Por ejemplo, Hill y Laufer (2003) en un estudio con alumnos chinos de inglés L2 encontraron que las actividades de poslectura, enfocadas de manera explícita en las palabras desconocidas, favorecían el aprendizaje por sobre las preguntas de comprensión relativas al significado de las palabras. Por otra parte, Paribakht y Wesche (1997) compararon directamente la lectura completamente incidental con la lectura acompañada de ejercicios de vocabulario. Los participantes de este estudio eran 38 hablantes nativos de diversas lenguas que tenían un nivel intermedio de inglés. En la condición de lectura incidental, los sujetos debían leer textos donde se presentaban palabras desconocidas, luego responder preguntas de comprensión lectora, para finalmente leer otros textos donde nuevamente aparecían las palabras nuevas. En la condición de lectura y ejercicios, los individuos leían textos equivalentes a los de la condición anterior y respondían preguntas de comprensión, pero en vez de leer nuevos textos, llevaban a cabo ejercicios de vocabulario. Los resultados de esta investigación demostraron que en ambas condiciones los participantes presentaron un avance significativo, pero el segundo tratamiento (lectura + ejercicios) mostraron un mejor desempeño. Similares resultados se encontraron en otra investigación donde se compararon dos grupos de estudiantes, cuyo origen también era diverso (Zimmerman, 1997). Esta investigadora descubrió que el grupo de estudiantes que desarrolló ejercicios interactivos de vocabulario después de la lectura mejoró de manera significativa en comparación al grupo que solo leyó los textos. Estudios más recientes han confirmado los hallazgos de las investigaciones pioneras en L2, utilizando diversas actividades centradas en el vocabulario, tales como ejercicios de comprensión lectora, ejercicios de vocabulario contextualizados, compleción de palabras, entre otras (Kim, 2008; Min, 2008; Peters, Hulstijn, Sercu \& Lutjeharms, 2009; Sonbul \& Schmitt, 2010; Little \& Kobayashi, 2011).

Una de las críticas a los estudios iniciales sobre aprendizaje a través de la lectura (Zimmerman, 1997; Paribakht \& Wesche, 1997) apunta a la falta de sensibilidad de las evaluaciones en las etapas iniciales del aprendizaje; por ejemplo, la asociación entre la forma ortográfica y el significado. Por esta razón, algunas investigaciones más recientes han propuesto medidas más efectivas para abordar el problema relacionado 
el aprendizaje léxico inicial. En esta línea, Sonbul y Schmitt (2010) decidieron utilizar nuevas pruebas que permitían evaluar de manera más eficaz la producción de la forma ortográfica, la producción del significado y el reconocimiento del significado. En un estudio experimental que tuvo un diseño muy similar a los presentados anteriormente, se evaluó el aprendizaje de vocabulario en la condición de lectura incidental y lectura acompañada de una explicación directa de los significados. Las participantes incluían 40 estudiantes universitarias de Arabia Saudita, quienes debieron aprender palabras nuevas en ambas condiciones experimentales. Al término de la etapa de aprendizaje, las participantes fueron evaluadas utilizando una prueba que comprendía tres actividades: compleción de palabras, traducción en L1 y selección múltiple. El análisis de los datos demostró que las estudiantes obtuvieron mejores resultados en la condición de lectura incidental con explicación directa de significados en las tres tareas, es decir, presentaron una mejor producción de la forma ortográfica, además de una mejor producción y reconocimiento del significado.

En el estudio de Sonbul y Schmitt (2010) nuevamente se confirma la superioridad de la lectura acompañada de actividades de instrucción explícita por sobre la lectura incidental. Sin embargo, a pesar de evaluar con mayor precisión el aprendizaje léxico en una etapa inicial, los investigadores no controlaron el tiempo de exposición que tuvieron las participantes a ambas condiciones, pues la lectura con instrucción explícita necesariamente incurrió en un mayor tiempo que la lectura en solitario. Esto hace difícil apreciar el real beneficio de la instrucción explícita, ya que su efecto podría ser atribuible simplemente a otras variables, tales como el tiempo de trabajo o la atención durante la tarea. Por otra parte, si bien todos los estudios presentados anteriormente utilizaron una amplia gama de pruebas para evaluar diferentes aspectos del aprendizaje léxico, ninguno de ellos empleó métodos que permitan medir con precisión el tiempo de procesamiento de las palabras adquiridas. Debido a que el aprendizaje completo de un ítem léxico toma un tiempo considerable y un número de exposiciones aún incierto (Webb, 2008), la utilización de tareas psicolingüísticas para medir el aprendizaje en etapas tempranas cobra un sentido fundamental. Estas podrían incluir lectura en voz alta, decisión léxica o categorización semántica y permitirían formular planteamientos sobre los factores que intervienen en el acceso léxico.

Como se explicó al comienzo de este apartado, la semántica juega un papel importante en el procesamiento de las palabras, ya que permite un acceso léxico más eficiente y más rápido en diferentes tareas psicolingüísticas incluyendo decisión léxica y categorización semántica (ver Ferreira et al., en prensa; Hargreaves \& Pexman, 2012; Pexman et al., 2013). El rol de la semántica en el acceso al léxico es uno de los aspectos psicolingüísticos que se abordan en la discusión de los resultados de este trabajo y que permite entregar planteamientos claros sobre los aspectos cognitivos de las palabras aprendidas recientemente en una lengua extranjera.

Es importante destacar que los estudios sobre aprendizaje léxico en L2 comúnmente no ofrecen explicaciones cognitivas de cómo se aprenden palabras y por qué la presentación de información explícita produce un mejor aprendizaje. 
En uno de los pocos trabajos donde se han abordado los aspectos psicolingüísticos del aprendizaje contextual y el rol de las actividades explícitas en la adquisición de palabras nuevas, Bolger et al. (2008) propusieron la teoría de instanced-based learning para explicar la influencia del contexto y el uso de definiciones en el aprendizaje del significado de las palabras. Los investigadores señalan que la aparición de una palabra en múltiples contextos (como en el caso de la lectura) permite generar la 'abstracción' de su significado. Esto se produce debido a que cada contexto nuevo representa una nueva huella de memoria episódica que, en combinación con otras, genera un concepto independiente de los eventos individuales. Por otra parte, la inclusión de definiciones o, entiéndase, cualquier otra actividad explícita de instrucción directa, permitiría tener un acceso más rápido al significado central (core) del léxico; es decir, al concepto que se genera producto de la exposición a diferentes contextos o episodios. Otra visión alternativa para explicar este último fenómeno corresponde a la involvement load hypothesis planteada por Hulstijn y Laufer (2001) y posteriormente extendida por el trabajo de (Kim, 2008). De acuerdo a esta hipótesis, mientras más esfuerzo requiere una tarea durante su ejecución mayor es el grado de retención o aprendizaje que se logra. Este planteamiento ha permitido explicar por qué actividades que van más allá de la simple lectura de un texto permiten generar un aprendizaje más eficiente y duradero.

Teniendo en cuenta los hallazgos de las investigaciones presentadas anteriormente y las teorías que describimos en este apartado, en el presente trabajo se espera que los participantes tengan un mejor desempeño en la condición LEV que en la condición LI, tanto en la tarea de reconocimiento visual como en la de categorización semántica. Debido a la falta de estudios experimentales que permitan explicar con claridad los procesos cognitivos que sirven de soporte al aprendizaje léxico en L2, en la presente investigación no solo se busca someter a prueba las hipótesis planteadas previamente, sino que también se ofrece una aproximación psicolingüística al aprendizaje léxico en L2. En particular, se pretende abordar cómo se accede al léxico nuevo y de qué forma la lectura y las actividades explícitas afectan el procesamiento de este en tareas de reconocimiento visual y categorización semántica.

\section{Marco metodológico}

\subsection{Participantes}

La muestra estuvo conformada por 20 alumnos (11 niñas y 9 niños) de sexto básico de un colegio privado de la ciudad de Concepción. Antes de seleccionar a los participantes, se aplicó un test de entrada KET (Cambridge English, 2013) a todos los alumnos de sexto básico (31 en total). Después de conocer los resultados del test, solo los alumnos que obtuvieron un puntaje promedio de 70 puntos o más (de un máximo de 100) fueron seleccionados para participar en el estudio. Debido a que todos ellos eran menores de edad, se debió obtener un consentimiento informado por parte de los padres con anterioridad al comienzo del experimento. 


\subsection{Materiales y diseño del estudio}

El presente estudio corresponde a un diseño experimental de medidas repetidas que permitió comparar el efecto de LI y LEV en el aprendizaje léxico con la utilización de las tareas de reconocimiento visual y categorización semántica. Se utilizaron 28 seudopalabras (p. ej., balter, gink) que fueron seleccionadas a partir de una serie de criterios en la página de The English Lexicon Project (http://elexicon.wustl.edu) (Balota, Yap, Hutchison, Cortese, Kessler, Loftis, Neely, Nelson, Simpson \& Treiman, 2007). Estos incluían longitud (4-6 letras), vecindad ortográfica (6-11 vecinos ortográficos), frecuencia de bigrama promedio (2.036-3.802), y tiempos de reacción (655-1050ms) y precisión (0.17-0.97) en tareas de decisión léxica. Al mismo tiempo, se seleccionaron otras 42 seudopalabras equivalentes a las utilizadas en el experimento que cumplían la función de distractores en los ejercicios de vocabulario de la condición LEV. Las 28 seudopalabras que los sujetos debían aprender se repartieron en dos series de 14 palabras cada uno. Con el fin de controlar todas las variables descritas anteriormente, se utilizó la prueba no paramétrica U de Mann-Whitney que permitió comparar las seudopalabras asignadas a cada condición en todas las variables. La prueba no mostró diferencias significativas en ninguna variable al comparar las dos series de palabras (longitud, $\mathrm{U}=98,00, p=1,00$; vecindad ortográfica, $\mathrm{U}=91,50, p=0,76$; frecuencia de bigrama, $\mathrm{U}=84,00 p=0,52$; Tiempos de reacción, $\mathrm{U}=90,00, p=0,71$; precisión, $\mathrm{U}=80,00, p=0,42)$. De esta forma, se puede afirmar que los ítems de cada serie eran equivalentes.

Después de este procedimiento, las 28 seudopalabras fueron distribuidas en 14 cuentos creados especialmente para el estudio, a partir de historias infantiles encontradas en diversos sitios de Internet. La mitad de las seudopalabras fue asociada a conceptos que correspondían a animales (p. ej., dax), mientras que la otra mitad fue asociada a objetos (p. ej., hassock). A través de una encuesta, se determinó que los participantes en este estudio desconocían el nombre real de estos conceptos aunque estaban familiarizados con sus rasgos visuales, puesto que conocían otros animales u objetos con características similares. Los cuentos tenían en promedio 207 palabras $(\mathrm{DE}=43,7)$ y cada uno contenía dos seudopalabras, las cuales se repetían seis veces. Con el fin de controlar la longitud y el contenido de los textos, utilizamos la herramienta VocabProfile del Compleat Lexical Tutor v.6.2 (Cobb, 2005) para obtener las medidas de las palabras K1 (alta frecuencia), K2 (frecuencia media) y AWL (académicas), además de la cantidad de palabras funcionales (verbos) y de contenido (pronombres y sustantivos). Después de este procedimiento, se aplicó la prueba no paramétrica U de Mann Whitney con el fin de comparar los textos de ambas condiciones en todas las variables descritas precedentemente. No se encontraron diferencias significativas entre los textos en ninguna de las variables $(K 1, \mathrm{U}=24,00, p=0,42 ; \mathrm{K} 2, \mathrm{U}=25,00$, $p=0,46$; AWL, $\mathrm{U}=20,00, p=0,12$; palabras funcionales, $\mathrm{U}=23, p=0,34$; palabras de contenido, $\mathrm{U}=17,50, p=0,12$ ). Después de haber controlado todas las variables lingüísticas necesarias, cada uno de los textos fue leído y editado por dos profesores, 
hablantes nativos de inglés, con el fin de validar el correcto uso de la lengua y el estilo en los cuentos para niños. Los profesores llevaron a cabo el proceso de validación por separado, pero no hubo diferencias sustanciales en las ediciones de cada uno, por lo que fue relativamente fácil incorporar los cambios de ambos docentes en un mismo escrito.

Tanto en la condición LEV como en la condición LI, se utilizaron ocho cuentos, de los cuales cuatro hacían referencia a animales y cuatro a objetos, y contenían 16 seudopalabras en total. Se redactaron 16 preguntas de comprensión lectora (dos por cada cuento) que no mencionaban las seudopalabras ni se requería de ellas para llevar a cabo las tareas del experimento (p. ej., Why does Emma love Christmas?). El objetivo de las preguntas de comprensión lectora era simplemente asegurarse de que los participantes mantuvieran la atención durante la etapa de aprendizaje. En la condición LI los participantes debían leer dos veces los cuentos. Sin embargo, en la condición LEV solo debían leerlos una vez y luego llevar a cabo cinco ejercicios de vocabularios que incluían las 28 seudopalabras: 1. Leer la descripción y encerrar el objeto/animal apropiado; 2. Elegir la frase que mejor describía el objeto/animal: 3. Unir el objeto/animal con la descripción; 4. Crucigrama; y 5. Sopa de letras. Los ejercicios no presentaban información adicional sobre las palabras nuevas ni requerían más tiempo que la segunda lectura en la condición LEV. Por otra parte, la frecuencia de aparición de las palabras fue la misma tanto en la condición LI como en LEV.

\subsection{Procedimiento}

El experimento se llevó a cabo en una sala aledaña a la biblioteca del colegio que reunía las condiciones necesarias para conducir la investigación. Durante tres días seguidos los participantes completaron dos sesiones de aprendizaje, en los dos primeros días, y una de evaluación, en el tercer día. Cada sesión de aprendizaje duraba alrededor de 90 minutos aproximadamente y los tratamientos de cada condición fueron administrados con un orden de exposición contrabalanceado, con el fin de controlar el posible efecto de orden de presentación en el aprendizaje léxico. Antes de comenzar la primera sesión, los participantes fueron divididos en dos grupos aleatorios (grupo A y grupo B), con el fin de hacer posible el contrabalanceo para controlar el orden de exposición. De esta forma, en la primera sesión, el grupo A fue expuesto a la condición LI y el grupo B a la condición LEV. En la segunda sesión de aprendizaje, el orden de presentación fue inverso para ambos grupos. En las dos condiciones, los participantes recibían instrucciones orales sobre todas las actividades que debían completar, además de instrucciones escritas antes de comenzar cada sesión. Después de leer las instrucciones de la primera parte, se procedía a la entrega de los textos uno por uno, con el fin de evitar que los individuos leyeran los textos más de una vez.

Con el objetivo de verificar el nivel de comprensión de los textos y asegurarnos de que los participantes estuvieran realizando la tarea, se incluyeron 16 preguntas 
de comprensión lectora, al final de la etapa de lectura, que no mencionaban las seudopalabras. En el segundo día de aprendizaje, los individuos debían leer nuevamente los mismos textos, en la condición LI, mientras que en la condición LEV, se les entregaban ejercicios de vocabulario donde aparecían las palabras nuevas. En la primera actividad, los participantes debían leer una definición y luego seleccionar la palabra que correspondía a esa definición de una lista de cuatro posibles opciones; tres eran seudopalabras que los sujetos nunca habían visto. La segunda actividad consistía en elegir la frase que mejor describía al objeto o animal a partir de tres opciones, donde solo una correspondía a la respuesta correcta mientras las otras dos entregaban información no relacionada con el significado de la palabra crítica. El tercer ejercicio requería que los participantes simplemente unieran las palabras nuevas en una columna con las descripciones de estas en otra columna. En la cuarta actividad se entregaron diferentes descripciones enumeradas y los sujetos debían completar un crucigrama con las palabras nuevas que correspondían a esas definiciones. Finalmente, al igual que en el ejercicio anterior, se entregó una lista de definiciones, pero los participantes debían encontrar las palabras nuevas en una sopa de letras. En total, los sujetos fueron expuestos 12 veces a cada palabra en cada condición y en un lapsus de tiempo equivalente.

En el tercer día, se aplicaron las pruebas psicolingüísticas de reconocimiento visual y categorización semántica, con la utilización de un computador portátil y el software DMDX (Forster \& Forster, 2003). En la prueba de reconocimiento visual, las 28 palabras fueron exhibidas de manera aleatoria en letra Times New Roman, minúscula, negrita y de tamaño 20. Antes de llevar a cabo cada una de las tareas del experimento, los participantes debían realizar una sesión de práctica idéntica a la prueba real, la cual incluía palabras familiares y tenía como fin entender la tarea para evitar errores involuntarios en el inicio. En la tarea de reconocimiento visual, la presentación de las palabras críticas comenzaba con la aparición de una cruz de fijación (+) en la pantalla durante $1000 \mathrm{~ms}$, seguida de la palabra aprendida recientemente por $2000 \mathrm{~ms}$. Los participantes debían presionar la flecha izquierda del teclado si ellos recordaban haber visto la palabra en la sesión de aprendizaje, o la flecha derecha, si no recordaban haberla estudiado.

En la tarea de categorización semántica, cada una de las 28 palabras aprendidas aparecía en la pantalla del computador por un tiempo de $2.000 \mathrm{~ms}$ y en orden aleatorio. $\mathrm{Al}$ igual que en la tarea de reconocimiento visual, las palabras estaban precedidas por una cruz de fijación $(+)$ que permanecía en la pantalla durante $1.000 \mathrm{~ms}$. Los participantes debían presionar la flecha derecha del teclado si la palabra que veían en la pantalla correspondía a un ser vivo, o la flecha izquierda, si esta hacía referencia a un objeto. En ambas tareas, se les recordaba a los sujetos presionar las teclas lo más pronto posible, pero teniendo en cuenta que debían evitar cometer errores. 


\section{Resultados}

En la Tabla 1, se presentan los tiempos de reacción (correspondientes a respuestas correctas), el porcentaje de respuestas correctas y la desviación estándar para las dos condiciones experimentales en ambas tareas.

Tabla 1. Tiempos de reacción y porcentajes de precisión en las tareas de reconocimiento visual y categorización semántica.

\begin{tabular}{|c|c|c|}
\hline & \multicolumn{2}{|c|}{ Condiciones experimentales } \\
\hline & Lectura incidental & Lectura + ejercicios de vocabulario \\
\hline TRs & \multicolumn{3}{|c|}{ Reconocimiento visual } \\
\hline$D E$ & 246 & 937 \\
\hline$\% \mathrm{RCs}$ & 39 & 186 \\
\hline & \multicolumn{3}{|c|}{ Categorización semántica } \\
\hline TRs & 1249 & 1186 \\
\hline$D E$ & 212 & 203 \\
\hline$\% \mathrm{RCs}$ & 64 & 75 \\
\hline
\end{tabular}

Nota: TRs, tiempos de reacción; DE, desviación estándar; RCs, respuestas correctas.

El análisis de los datos emitidos por los 20 participantes en ambas tareas fue realizado utilizando una prueba $t$ de Student para muestras relacionadas. El análisis fue aplicado a los tiempos de reacción y a los porcentajes de respuestas correctas.

En la tarea de reconocimiento visual, se encontró una diferencia significativa entre las condiciones LEV y LI, $t_{1}(19)=2,62, p=0,02 ; d=0.46$. Esto significa que el tiempo de reacción de los participantes en decidir si habían visto las palabras aprendidas recientemente fue significativamente menor en la condición LEV que en la condición LI. Al igual que en los TRs, el análisis estadístico del porcentaje de respuestas correctas encontró una diferencia significativa entre las condiciones LEV y LI, $t_{1}(19)=9,06, p<0,001 ; d=2.61$ con un porcentaje mayor de respuestas correctas en la condición LEV (ver Gráfico 1). 


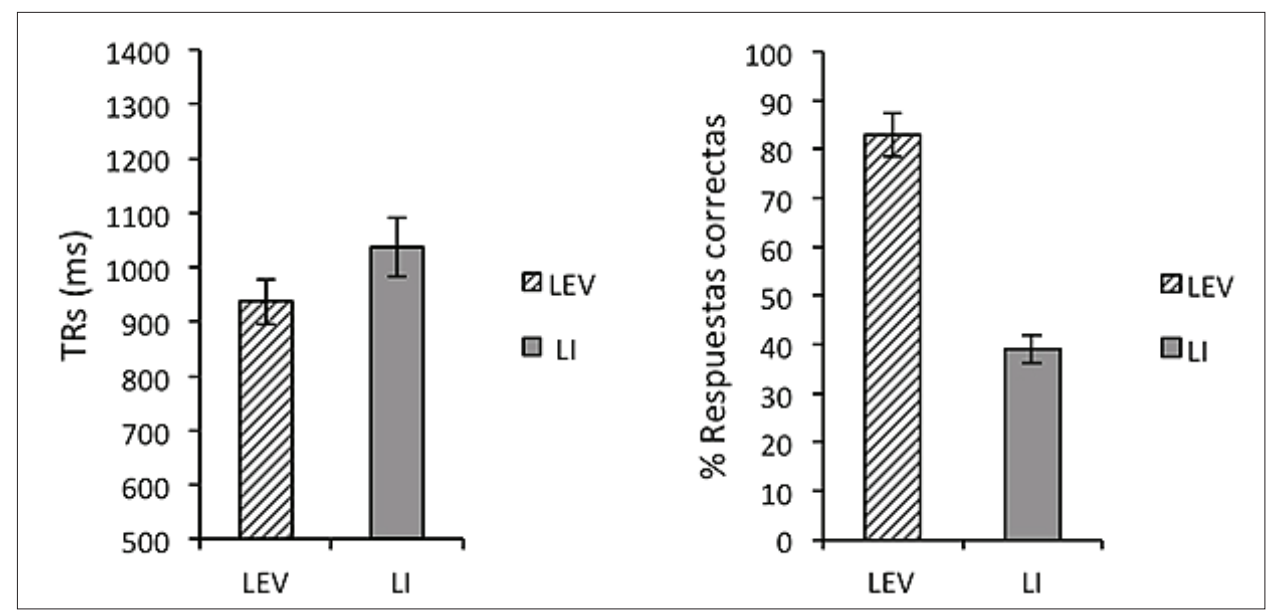

Nota: TRs, tiempos de reacción; DE, desviación estándar; RCs, respuestas correctas. Las barras de error representan el error estándar.

Gráfico 1. TRs y CRs en la tarea de reconocimiento visual en las condiciones LEV y LI.

En la categorización semántica, no se encontraron diferencias significativas entre las condiciones LEV y LI para el análisis de TRs, $t 1(19)=1,70, p=0,11 ; d=0.30$. Sin embargo, hubo una diferencia altamente significativa en el análisis del porcentaje de respuestas correctas, $t 1(19)=3,02, p=0,01 ; d=0.91$, donde la condición LEV obtuvo un mayor porcentaje en comparación con la condición LI (ver Gráfico 2).

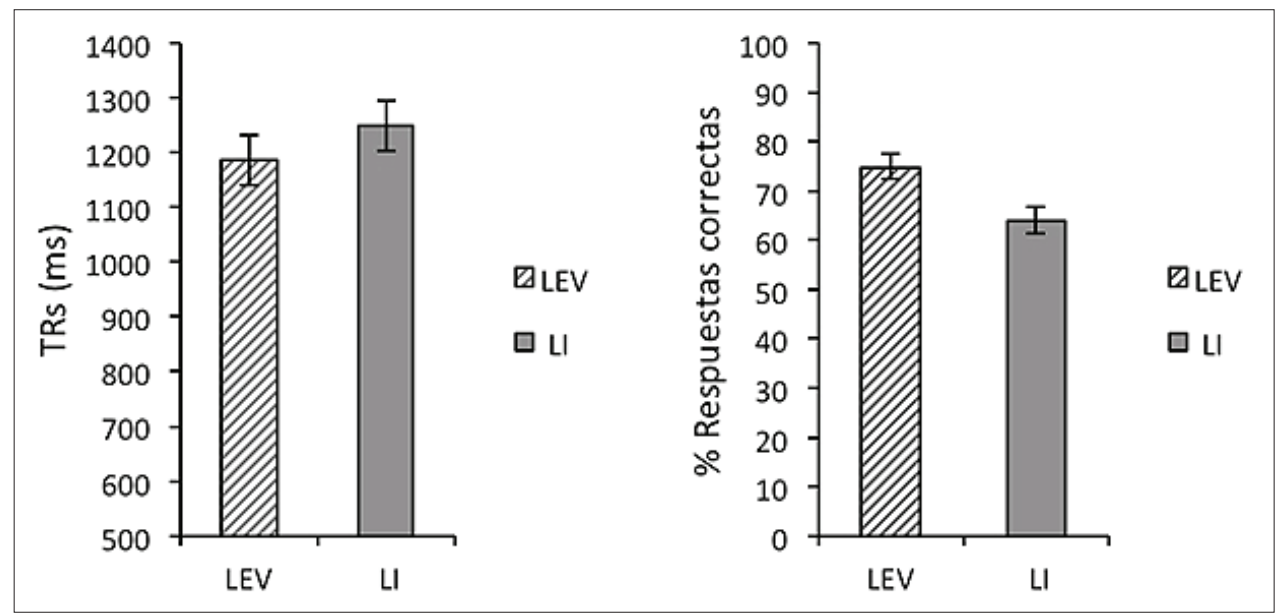

Nota: TRs, tiempos de reacción; DE, desviación estándar; RCs, respuestas correctas. Las barras de error representan el error estándar.

Gráfico 2. TRs y CRs en la tarea de categorización semántica en las condiciones LEV y LI. 


\section{Discusión}

En este trabajo se buscaba, en primer lugar, dilucidar si la lectura acompañada de ejercicios de vocabulario genera un mayor nivel de aprendizaje que la lectura completamente incidental en las tareas receptivas de reconocimiento visual y categorización semántica. En segundo lugar, en esta investigación se intentaba entregar una explicación psicolingüística al proceso de aprendizaje contextual en L2 y al impacto que genera la introducción de actividades explícitas centradas en el léxico.

Los resultados de este estudio demostraron que el uso de la lectura incidental complementada con diversos ejercicios de vocabulario (LEV) produce menos errores y tiempos de reacción más rápidos que la lectura incidental (LI) en el proceso de reconocimiento visual de palabras nuevas. Por otra parte, el resultado en la prueba de categorización semántica también arrojó un menor número de errores en la condición LEV, pero no se encontraron diferencias significativas entre las condiciones con respecto a los tiempos de reacción. El efecto en esta última medida es prácticamente irrelevante dado que las diferencias en el número de errores fueron significativas. A nivel global, estos resultados confirman las hipótesis iniciales y señalan que la lectura por sí sola favorece el aprendizaje del léxico. Sin embargo, cuando esta se acompaña de ejercicios de vocabulario - sin necesidad de añadir más tiempo de aprendizaje o mayor frecuencia de presentación-genera mejores resultados en tareas receptivas (reconocimiento visual y categorización semántica).

Dado lo anterior, podemos aseverar que nuestra investigación está en línea con los resultados de estudios previos sobre el aprendizaje léxico y, además, aporta datos obtenidos a partir de un paradigma psicolingüístico. Al igual que en investigaciones anteriores (Zimmerman, 1997; Paribakht \& Wesche, 1997; Hill \& Laufer, 2003; Yali, 2010; Sonbul \& Schmitt, 2010; Little \& Kobayashi, 2011; Sharafi-Nejad, Raftari, Bijami, Khavari, Ismail \& Eng, 2014), hemos demostrado que la lectura incidental acompañada de actividades que permitan un acceso explícito a la forma visual y al significado de las palabras producen un mejor aprendizaje de la ortografía y la semántica del léxico.

A diferencias de los trabajos citados anteriormente, nuestro estudio tuvo un mayor control sobre las variables socioculturales, pues todos los participantes provenían de un mismo país y tenían como lengua materna el español. Por otra parte, la medición de tiempos de reacción, además del porcentaje de respuestas correctas en ambas tareas, ha conferido una mayor precisión a los efectos que se obtuvieron con respecto a estudios donde solo se ha contabilizado el número de respuestas correctas. Esta diferenciación es particularmente clara con respecto a los trabajos de Paribakht y Wesche (1997) y Zimmerman (1997), quienes no utilizaron tareas apropiadas para etapas iniciales de aprendizaje léxico. Finalmente, una ventaja comparativa con respecto al estudio de Sonbul y Schmitt (2010) radica en el hecho de que estos investigadores no controlaron de manera adecuada el tiempo de exposición de las condiciones, lo que dificulta la 
interpretación de sus resultados. Nuestro experimento ha permitido corroborar los descubrimientos de investigaciones precedentes, con la adición de conceder un mayor control al diseño y una mayor precisión a las medidas de los efectos.

Además de las ventajas comparativas de nuestro paradigma, en esta investigación nos propusimos avanzar hacia una explicación de los aspectos cognitivos que subyacen a la adquisición del léxico en L2. Si bien el aprendizaje contextual o aprendizaje a través de la lectura incidental ha sido investigado en innumerables oportunidades, la gran mayoría de los estudios no han explicado con claridad cómo accedemos al significado de las palabras o cómo aprendemos a reconocer su forma visual en un texto. Por otra parte, tampoco se ha abordado la razón por la cual las actividades explícitas mejoran el desempeño en el aprendizaje léxico. Uno de los pocos estudios hasta ahora que ha intentado abordar los aspectos cognitivos del aprendizaje contextual ha sido el de Bolger et al. (2008). Este trabajo se apoya en el modelo de memoria instance-based de Reichle y Perfetti (2003) para explicar cómo adquirimos el léxico a través de la lectura en una lengua materna. De acuerdo a este enfoque, cada vez que encontramos una palabra desconocida en un contexto determinado, se genera una huella de memoria episódica y, a su vez, se reactivan otras huellas de memoria asociadas a la palabra. De esta forma encontrar una palabra nueva en diferentes contextos permite la generación y reactivación de diferentes huellas de memoria que hacen posible la abstracción del significado y el almacenamiento de un nuevo ítem léxico independiente del contexto.

En nuestro estudio, los participantes fueron expuestos a cada palabra de manera incidental al menos 6 veces (12 en la condición completamente incidental), lo que nos permite aseverar que generaron algún nivel de abstracción del significado, incluso en la condición que presentó los resultados más deficientes. De lo contrario, los participantes del estudio no habrían obtenido resultados por sobre el nivel de base (50 por ciento) en la tarea de categorización semántica.

El modelo de aprendizaje instanced-based también entrega un indicio del aporte de las actividades explícitas al aprendizaje léxico. Bolger et al. (2008) sostienen, por ejemplo, que el uso de definiciones es efectivo porque pueden interactuar con el contexto y así comunicar el significado abstracto de las palabras de manera más directa. Esto es posible debido a que las definiciones son contextos específicos que contienen rasgos semánticos centrales y, por lo tanto, tienen el potencial de asociarse más fácilmente con los nuevos contextos en los que aparece una palabra. En la presente investigación, podemos extender este planteamiento y afirmar que no solo las definiciones son contextos que permiten acelerar el aprendizaje, sino que cualquier actividad explícita de vocabulario que comunique los atributos centrales del léxico. Por esta razón, los ejercicios de vocabulario que utilizamos son ciertamente comparables a las definiciones, ya que requerían comparar o evaluar los atributos más prominentes de las palabras que estaban en proceso de aprendizaje. De esta forma, los mejores resultados en la condición LEV en ambas tareas se deberían a una más rápida abstracción del léxico y, en consecuencia, una mayor integración del significado. 
Una explicación alternativa al modelo instanced-based para explicar el avance que experimentaron los participantes en la condición LEV podría ofrecerse desde la teoría de testing effect (Tulving, 1967; Roediger \& Karpicke, 2006). Esta teoría señala que una instancia de prueba permite generar un mejor aprendizaje en comparación a presentar un mismo contenido nuevamente. En consecuencia, tomar un test de memoria no solo mide lo que sabemos, sino que también mejora la retención del conocimiento adquirido. En la presente investigación, los cinco tipos de ejercicios de vocabulario que debieron realizar los participantes en la condición LEV pueden ser considerados una instancia de prueba del conocimiento, pues requerían que los sujetos recordaran las palabras nuevas que habían experimentado a través de la lectura incidental. Este proceso, que involucra trabajar con las palabras inmediatamente después de la lectura, facilitaría el aprendizaje. En esta misma línea, Paribakht y Wesche (1997) sugieren que encontrarse con las palabras en múltiples ocasiones durante la lectura dirige a un cierto tipo de aprendizaje, pero el hacer un trabajo enfocado en el nuevo vocabulario demandaría un análisis más profundo, además de entregar una práctica extensiva, lo que proporcionaría un aprendizaje léxico más efectivo. Este planteamiento también concuerda con la involvement load hypothesis que hace referencia a los niveles cognitivos de procesamiento (Hulstijn \& Laufer, 2001). Los ejercicios de vocabulario sin duda requieren de un mayor esfuerzo que la lectura incidental, pues fuerzan al individuo a acceder al léxico y activar la información semántica de las palabras, además de sus conexiones con otras palabras en el lexicón mental. Este mayor esfuerzo en el procesamiento generaría un aprendizaje léxico más efectivo y más duradero (Kim, 2008).

En los párrafos anteriores se entregan algunas posibles explicaciones que permiten entender de mejor forma por qué las actividades explícitas centradas en el vocabulario generan un mejor aprendizaje. Esto representa un avance con respecto a la gran mayoría de los estudios en esta línea donde no se explican los procesos psicolingǘsticos inherentes al léxico y su adquisición. Otro aspecto importante de clarificar es lo referente específicamente a las tareas psicolingüísticas que se utilizaron. La investigación actual, además de evaluar el aprendizaje en las condiciones LEV y LI, también buscaba entender en profundidad qué significa que la condición LEV genere un mayor rendimiento en reconocimiento visual y categorización semántica. Se plantean dos posibles explicaciones apoyadas por la evidencia empírica de dos variables de uso recurrente en psicolingüística: la riqueza semántica y la diversidad contextual. Una gran cantidad de estudios han demostrado que la semántica juega un papel importante en el reconocimiento visual de las palabras. En particular, el proceso de reconocimiento visual se ve facilitado en el caso de las palabras con mayor riqueza semántica (Pexman, 2012). La evidencia empírica existente apunta a que se accede al significado de una palabra antes de que su forma ortográfica sea completamente identificada, posiblemente a través de una retroalimentación de activación semántica hacia las unidades ortográficas y fonológicas (Pexman, 2012; Yap, Pexman, Wellsby, Hargreaves \& Huff, 2012). Evidentemente la riqueza semántica no solo favorece tareas 
de reconocimiento visual (lectura en voz alta, decisión léxica), sino que su influencia también puede apreciarse en una serie de otras tareas psicolingüísticas, por ejemplo, la categorización semántica. Ferreira et al. (en prensa) investigaron el efecto de la riqueza semántica tanto a nivel conductual como neuronal y encontraron que las palabras (familiares y aprendidas recientemente) con mayor cantidad de atributos semánticos se clasificaban con mayor grado de precisión y generaban tiempos de reacción más rápidos. Por otra parte, la riqueza semántica también produjo efectos notables a nivel neuronal donde las palabras con mayor número de atributos semánticos mostraron más activación en zonas involucradas en la representación semántica del léxico (p. ej., los giros temporal medio y angular del hemisferio izquierdo). Es posible que en el presente trabajo los ejercicios de vocabulario, producto de un mayor esfuerzo de procesamiento, generen un aprendizaje más significativo que constituya una riqueza semántica más amplia que en la condición de solo lectura. El hecho de que las palabras tengan una mayor riqueza semántica permitiría generar mejor desempeño tanto en reconocimiento visual como en categorización semántica, en línea con los estudios previos citados en este apartado.

Otro factor que podría estar detrás del mayor aprendizaje que genera la lectura con ejercicios de vocabulario es la diversidad contextual. El estudio de esta variable es relativamente nuevo y tuvo su origen en el trabajo de Adelman et al. (2006) quienes demostraron que la diversidad contextual podía predecir de mejor forma el éxito en las tareas de lectura y decisión léxica que la frecuencia. Además, la diversidad contextual también se ha definido como un factor relevante en la adquisición léxica por parte de los niños cuando estos están aprendiendo a leer (Hills, Maouene, Riordan \& Smith, 2010). En esta misma línea, Perea et al. (2013) también encontraron que esta variable determina el tiempo que toma un niño en identificar una palabra en una tarea de decisión léxica. De acuerdo a Adelman et al. (2006), la ventaja de las palabras que aparecen en contextos diversos se debe a que estos facilitan su accesibilidad en el lexicón mental. En el caso de las palabras que han sido aprendidas recientemente, estas formarían mayores conexiones con otras palabras en el lexicón mental y, de esta forma, se activarían de manera más eficiente al ser evocadas en tareas de reconocimiento visual o semánticas. En el caso particular del presente estudio, es factible plantear que las palabras que se enseñan con la adición de ejercicios de vocabulario tienen mayor número de contextos, pues cada ejercicio nuevo representa no solo la oportunidad de ver nuevamente una palabra, sino también de observarla en un contexto distinto.

\section{CONCLUSIONES}

El presente trabajo ha permitido corroborar los descubrimientos de investigaciones previas sobre la eficacia de la lectura acompañada de actividades explícitas en la adquisición del léxico en L2. Además, la formulación de un mayor control sobre las variables socioculturales y psicolingüísticas ha permitido avanzar hacia una teoría psicolingüística del aprendizaje léxico contextual y la contribución 
del uso de actividades explícitas en L2. Las investigaciones anteriores en esta línea se habían limitado a describir el efecto de los factores que aquí se estudiaron, pero no habían abordado los procesos cognitivos que subyacen a estos. En suma, esta investigación aporta una nueva perspectiva al estudio del aprendizaje contextual y abre oportunidades para más estudios que se propongan profundizar en esta área.

Un punto de partida para nuevas investigaciones podría darse en el plano de la producción léxica. Este estudio se centró en medir el aprendizaje léxico utilizando dos tareas receptivas (reconocimiento visual y categorización semántica). Esto implica que no se evaluó si los participantes eran capaces de producir las palabras aprendidas, de mediar un contexto que las evocara. De esta forma, un estudio futuro en esta línea podría abordar en qué medida este paradigma permite alcanzar niveles de producción léxica. Para esto, se podrían incluir actividades que precisamente requieran producir vocablos, como el nombrado de imágenes (una imagen aparece en la pantalla del computador y el participante debe decir el nombre de esta), recuerdo guiado (se presentan las primeras letras de una palabra y el sujeto debe escribir las letras que faltan) o compleción de oraciones (mostrar oraciones que no contengan la palabra crítica para que el/la participante la escriba). Otros factores que pueden investigarse a la par con la producción léxica serían la cantidad de exposiciones, la calidad del contexto y el tipo de actividad explícita poslectura. En diseños experimentales más complejos que el nuestro podría observarse la forma en que estos factores interactúan en el proceso de adquisición léxica con el fin de formular una teoría psicolingüística más específica del aprendizaje léxico contextual y el aporte de las actividades explícitas en L2.

Finalmente, es necesario hacer un alcance sobre la forma en que este trabajo podría entregar lineamientos a posibles aplicaciones en el aula. A partir de nuestros resultados y los de otras investigaciones en la misma línea, podemos afirmar que la lectura es una fuente importante de adquisición léxica y que los ejercicios de vocabulario permiten generar un mejor aprendizaje. De esta forma, recomendamos el uso de una metodología que entregue la oportunidad a los alumnos de leer textos adecuados a su nivel y que desarrollen conjuntamente actividades explícitas para reforzar el aprendizaje del léxico desconocido. Por otra parte, sería importante tener presente que el aprendizaje incidental es un proceso lento que requiere múltiples instancias de exposición, por lo que es necesario diseñar diferentes ejercicios de vocabulario complementarios que tengan en consideración la diversidad contextual y la riqueza semántica del léxico, además de evaluaciones adecuadas para las diferentes instancias y niveles de aprendizaje. 


\section{REFERENCIAS BIBLIOGRÁFICAS}

Adelman, J. S., Brown, G. D. \& Quesada, J. F. (2006). Contextual diversity, not word frequency, determines word-naming and lexical decision times. Psychological Science, 17(9), 814-823.

Ahmad, J. (2012). Intentional vs. incidental vocabulary learning. ELT Research Journal, 1(1), 71-79.

Balota, D. A., Yap, M. J., Hutchison, K. A., Cortese, M. J., Kessler, B., Loftis, B., Neely, J. H., Nelson, D. L., Simpson, G. B. \& Treiman, R. (2007). The English lexicon project. Behavior Research Methods, 39(3), 445-459.

Bannard, C. \& Matthews, D. (2008). Stored word sequences in language learning: The effect of familiarity on children's repetition of four-word combinations. Psychological Science, 19(3), 241-248.

Bolger, D. J., Balass, M., Landen, E. \& Perfetti, C. A. (2008). Context variation and definitions in learning the meanings of words: An instance-based learning approach. Discourse Processes, 45(2), 122-159.

Cambridge English. (2013). Cambridge English: Key (KET). [en línea]. Disponible en: http://www.cambridgeenglish.org/exams/key/.

Cobb, T. (2005). Compleat lexical tutor v.6.2. [en línea]. Disponible en: http://www. lextutor.ca/.

Ferreira, R. A. (2011). Learning the meaning of new words: Behavioural and neuroimaging evidence. Tesis doctoral, Universidad de York, York, Reino Unido.

Ferreira, R. A., Göbel, S., Hymers, M. \& Ellis, A. W. (en prensa). The neural correlates of semantic richness: Evidence from an fMRI study of word learning. Brain and Language.

Forster, K. I. \& Forster, J. C. (2003). DMDX: A Windows display program with millisecond accuracy. Behavior Research Methods, Instruments \& Computers, 35(1), 116-124.

Grabe, W. (2008). Vocabulary and reading comprehension. En W. Grabe (Ed.), Reading in a Second Language (pp. 265-286). Cambridge: Cambridge University Press.

Hargreaves, I. S. \& Pexman, P. M. (2012). Does richness lose its luster? Effects of extensive practice on semantic richness in visual word recognition. Frontiers in Human Neuroscience, 6, 234.

Hill, M. \& Laufer, B. (2003). Type of task, time-on-task and electronic dictionaries in incidental vocabulary acquisition. International Review of Applied Linguistics in Language Teaching, 41(2), 87-106. 
Hills, T. T., Maouene, J., Riordan, B. \& Smith, L. B. (2010). The associative structure of language: Contextual diversity in early word learning. Journal of Memory and Language, 63(3), 259-273.

Hulstijn, J. H. \& Laufer, B. (2001). Some empirical evidence for the involvement load hypothesis in vocabulary acquisition. Language Learning, 51(3), 539-558.

Jenkins, J. R., Matlock, B. \& Slocum, T. A. (1989). Two approaches to vocabulary instruction: The teaching of individual word meanings and practice in deriving word meaning from context. Reading Research Quarterly, 24(2), 215235.

Kim, Y. (2008). The role of task-induced involvement and learner proficiency in L2 vocabulary acquisition. Language Learning, 58(2), 285-325.

Kuppens, A. H. (2010). Incidental foreign language acquisition from media exposure. Learning, Media and Technology, 35(1), 65-85.

Lew-Williams, C. \& Fernald, A. (2007). Young children learning Spanish make rapid use of grammatical gender in spoken word recognition. Psychological Science, 18(3), 193-198.

Little, A. \& Kobayashi, K. (2011). Comparing listening and vocabulary enhancement activities for vocabulary acquisition and retention. The Malaysian Journal of ELT Research, 7(1), 1-63.

Min, H. T. (2008). EFL vocabulary acquisition and retention: Reading plus vocabulary enhancement activities and narrow reading. Language Learning, 58(1), 73-115.

Nagy, W. \& Anderson, R. C. (1984). How many words are there in printed school English? Reading Research Quarterly, 19(3), 304-330.

Nagy, W. \& Townsend, D. (2012). Words as tools: Learning academic vocabulary as language acquisition. Reading Research Quarterly, 47(1), 91-108.

Paribakht, T. S. \& Wesche, M. (1997). Vocabulary enhancement activities and reading for meaning in second language vocabulary development. En J. Coady \& T. Huckin (Eds.), Second language vocabulary acquisition: A rationale for pedagogy (pp. 174-200). Nueva York: Cambridge University Press.

Paribakht, T. S. \& Wesche, M. (1999). Reading and incidental L2 vocabulary acquisition. Studies in Second Language Acquisition, 21(2), 195-224.

Perea, M., Soares, A. P. \& Comesaña, M. (2013). Contextual diversity is a main determinant of word identification times in young readers. Journal of Experimental Child Psychology, 116(1), 37-44. 
Petchko, K. (2011). Input enhancement, noticing, and incidental vocabulary acquisition. The Asian EFL Journal Quarterly December, 13(4), 228-255.

Peters, E., Hulstijn, J. H., Sercu, L. \& Lutjeharms, M. (2009). Learning L2 German vocabulary through reading: The effect of three enhancement techniques compared. Language Learning, 59(1), 113-151.

Pexman, P. (2012). Meaning-based influences on visual word recognition. En J. S. Adelman (Ed.), Visual word recognition: Meaning and context, individuals and development (pp. 24-43). Hove, UK: Psychology Press.

Pexman, P. M., Siakaluk, P. D. \& Yap, M. J. (2013). Introduction to the research topic meaning in mind: Semantic richness effects in language processing. Frontiers in Human Neuroscience, 7, 723.

Rakoczy, H., Warneken, F. \& Tomasello, M. (2008). The sources of normativity: Young children's awareness of the normative structure of games. Developmental Psychology, 44(3), 875-881.

Rashidi, N. \& Ganbari-Adivi, A. (2010). Incidental vocabulary learning through comprehension focused reading of short stories. Journal of English Language Teaching and Learning, 53(2), 217-236.

Reichle, E. D. \& Perfetti, C. A. (2003). Morphology in word identification: A wordexperience model that accounts for morpheme frequency effects. Scientific Studies of Reading, 7(3), 219-237.

Roediger, H. L. \& Karpicke, J. D. (2006). Test-enhanced learning taking memory tests improves long-term retention. Psycbological Science, 17(3), 249-255.

Rott, S. (1999). The effect of exposure frequency on intermediate language learners' incidental vocabulary acquisition and retention through reading. Studies in Second Language Acquisition, 21(4), 589-619.

Sharafi-Nejad, M., Raftari, S., Bijami, M., Khavari, Z., Ismail, S. A. M. M. \& Eng, L. S. (2014). The impact of vocabulary enhancement activities on vocabulary acquisition and retention among male and female EFL learners in Iran. English Language Teaching, 7(4), 126-135.

Sonbul, S. \& Schmitt, N. (2010). Direct teaching of vocabulary after reading: is it worth the effort? ELT Journal, 64(3), 253-260.

Stahl, S. A. (1991). Beyond the instrumental hypothesis: Some relationships between word meanings and comprehension. En P. J. Schwanenflugel (Ed.), The Psychology of word meanings (pp. 157-185). Hillsdale, N.J.: Erlbaum.

Tulving, E. (1967). The effects of presentation and recall of material in free-recall learning. Journal of Verbal Learning and Verbal Behavior, 6(2), 175-184. 
Vidal, K. (2011). A comparison of the effects of reading and listening on incidental vocabulary acquisition. Language Learning, 61(1), 219-258.

Webb, S. (2007). The effects of repetition on vocabulary knowledge. Applied Linguistics, 28(1), 46-65.

Webb, S. (2008). The effects of context on incidental vocabulary learning. Reading in a Foreign Language, 20(2), 232-245.

Yali, G. (2010). L2 vocabulary acquisition through reading: Incidental learning and Intentional learning. Chinese Journal of Applied Linguistics, 33(1), 74-93.

Yap, M. J., Pexman, P. M., Wellsby, M., Hargreaves, I. S. \& Huff, M. J. (2012). An abundance of riches: Cross-task comparisons of semantic richness effects in visual word recognition. Frontiers in Human Neuroscience, 6, 72.

Zimmerman, C. B. (1997). Do reading and interactive vocabulary instruction make a difference? An empirical study. TESOL Quarterly, 31(1), 121-140.

\section{* AGRADECIMIENTO}

Esta investigación se realizó en el marco del proyecto Fondecyt 11130678, Word Learning in Bilinguals. 